\title{
Comparison of Mobile-Bearing and Fixed-Bearing Designs in High Flexion Total Knee Arthroplasty: Using a Navigation System
}

\author{
Tae Wan Kim, MD, Shi Hwan Park, MD and Jeung Tak Suh, MD, PhD \\ Department of Orthopaedic Surgery, Pusan National University School of Medicine, Busan, Korea
}

Purpose: We compared and analyzed the short term results of high flexion total knee arthroplasty (TKA) with mobile-bearing and fixed bearing designs.

Materials and Methods: We studied 32 patients that had undergone TKA with LPS-Flex Mobile and 34 patients with LPS-Flex Fixed using an electromagnetic navigation system between January 2010 and June 2010, and were followed up for at least 1 year.

Results: Knee Society Functional Score (KSFS) and Knee Society Knee Score (KSKS) of the mobile-bearing group were 94.5 and 93.8 points, respectively, and were 48.2 and 45.3 points preoperatively, whereas those of the fixed-bearing group were 95.1 and 94.2 points, respectively, and were 49.5 and 46.9 points preoperatively. Postoperative mechanical axis deviation and implant position of the femoral and tibial component both on the coronal and sigittal planes showed no significant differences between the two groups. Range of motion (ROM) and maximal flexion angle (MFA) of the knee joint also showed no significant differences between the two groups. The possibility of crossed-legged sitting and kneeling position also showed no significant differences between the two groups.

Conclusions: Clinical and radiologic parameters, ROM and MFA of knee joints showed no significant differences in both the groups, but long term follow-up results may be necessary, including survival rate.

Key words: Knee joint, High flexion total knee arthroplasty, Electromagnetic navigation system, Mobile-bearing, Fixed-bearing.

\section{Introduction}

The goals of total knee arthroplasty (TKA) are to relieve pain, maintain knee function and kinematics, and restore stability and range of motion (ROM) to facilitate daily activities. Of these, restoration of ROM has been considered important especially in Eastern countries where high flexion is required for religious

Received August 2, 2011; Revised (1st) August 23, 2011;

(2nd) October 4, 2011, 2011; Accepted October 17, 2011.

Correspondence to: Jeung Tak Suh, MD, PhD.

Department of Orthopaedic Surgery, Pusan National University School of Medicine, 179 Gudeok-ro, Seo-gu, Busan 602-739, Korea.

Tel: +82-51-240-7248, Fax: +82-51-247-8395

Email: jtsuh@pusan.ac.kr

This is an Open Access article distributed under the terms of the Creative Commons Attribution Non-Commercial License (http://creativecommons.org/licenses/by-nc/3.0/) which permits unrestricted non-commercial use, distribution, and reproduction in any medium, provided the original work is properly cited. and social activities. Factors that can be associated with ROM include the cause of disease, preoperative joint deformity and ROM, age, gender, surgical technique, implant fixation strength, postoperative rehabilitation, and implant design ${ }^{1)}$.

Since first introduced in TKA for optimal component positioning and lower limb alignment, navigation systems have been improved with rapid development of computer technology to allow for soft tissue balancing and flexion-extension gap balancing, and accordingly have contributed to the accuracy of $\mathrm{TKA}^{2)}$.

In this study, we analyzed the results of high flexion TKA using one of the most recently developed navigation systems, the electromagnetic navigation system, to compare the influence of two different implant designs (mobile-bearing and fixed bearing designs) on ROM including maximal flexion angle and clinical and radiographic results. The mobile-bearing implant used was NexGen LPS-Flex Mobile Knee (NexGen, Legacy PosteriorStabilized flexion Mobile, Zimmer, Warsaw, IN, USA) whereas the fixed-bearing implant was NexGen LPS-Flex Fixed Knee (NexGen, Legacy Posterior-Stabilized flexion Fixed, Zimmer). 


\section{Materials and Methods}

\section{Materials}

Of the patients that had undergone TKA by the same surgeon between January 2010 and June 2010 at our institution, 67 patients with a minimum follow-up of one year were randomly selected for this study. The implants used were the LPS-Flex Mobile knee in 32 patients and LPS-Flex Fixed knee in 34 patients. In six of the patients with bilateral TKA, the mobilebearing prosthesis was used on one side and the fixed-bearing on the other side. In all patients, an electromagnetic navigation system (Zimmer Computer Assisted Solutions Electromagnetic Quad-Sparing) was used. The mean follow-up period was 14.5 months (range, 12 to 18 months) and the mean age of the patients was 67.5 years (range, 60 to 78 years) in the mobile-bearing group and 68.5 years (range, 61 to 77 years) in the fixed-bearing group. The cause of injury was degenerative arthritis in all patients. The mean preoperative ROM was $118^{\circ}$ in both groups and no notable intergroup difference could be found regarding flexion contracture (Table 1).

Considering that the preoperative ROM could affect the postoperative $\mathrm{ROM}^{3,4)}$, we excluded patients who had $\leq 90^{\circ}$ of ROM preoperatively. There were no particular instructions for knee flexion exercises and the same rehabilitation protocol was prescribed in both groups.

\section{Clinical Assessment}

The clinical results were graded according to the Knee Society Knee Score (KSKS) and Knee Society Functional Score (KSFS). Patient satisfaction and preference were assessed according to the Western Ontario and McMaster Universities Arthritis Index (WOMAC) score ${ }^{5}$, a questionnaire for measuring pain, stiffness, and function. Postoperative pain was evaluated using the visual analog scale (VAS). A patient's ability to kneel and sit cross-legged

Table 1. Demographic Parameters of Both Treatment Groups in This Study

\begin{tabular}{lcc}
\hline & LPS-flex mobile & LPS-flex fixed \\
\hline Cases & 32 & 34 \\
Diagnosis & OA $(100 \%)$ & OA $(100 \%)$ \\
Sex (M:F) & $3: 29$ & $4: 30$ \\
Mean Age (yr) & 67.5 & 68.5 \\
Preoperative mechanical axis & Varus $7.0^{\circ}$ & Varus $7.4^{\circ}$ \\
Preoperative ROM $\left({ }^{\circ}\right)$ & $118.5(118.5 \pm 5.8)$ & $118.0(118.0 \pm 5.2)$ \\
Flexion contracture $\left({ }^{\circ}\right)$ & $3.5(3.5 \pm 1.6)$ & $3.8(3.8 \pm 1.5)$ \\
\hline
\end{tabular}

ROM: range of motion. was assessed through interview or observation during follow-up. Physical examination and goniometric measurement have been commonly used in the assessment of ROM and maximal flexion angle. However, we used a radiographic measurement method devised by Edwards et al. ${ }^{6}$ ) to reduce measurement errors and increase reproducibility. We measured the angle formed by lines drawn down the mid-shafts of the tibia and femur on the lateral radiographs taken with the knee in full extension and full flexion preoperatively, as well as at the final follow-up (Fig. 1).

\section{Radiographic Assessment}

Radiographic assessment was done by the same surgeon three times per patient and the mean value was used for analysis. The mechanical axis deviation was measured as the difference in the angle between the femoral mechanical axis and the tibial mechanical axis on the weight bearing long leg anteroposterior radiographs taken preoperatively and postoperatively. The femoral and tibial component positions in the sagittal and coronal planes were assessed using the method of Seon and Song $^{7}$. The coronal inclination of the femoral component was measured as the medial angle between the mechanical axis and the bottom of the component on the anteroposterior radiographs and the sagittal inclination as the angle with the femoral shaft on the lateral views. The desired sagittal inclination was defined as $90^{\circ}$. The coronal and sagittal tibial component positions were assessed in a similar manner. The bisecting line of the tibial shaft was used as a reference for sagittal tibial component positioning. The desired tibial component inclination was defined as $90^{\circ}$ in the coronal plane and $86^{\circ}$ in the sagittal plane (Fig. 2).

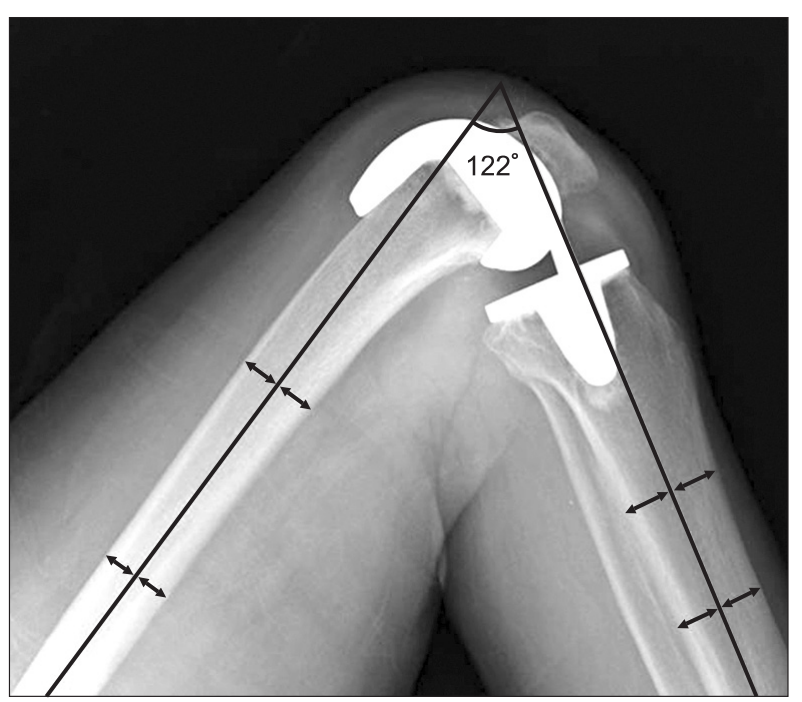

Fig. 1. Radiological measurements of mid-lines of the femur and tibia. 


\section{Surgical Technique}

All the operations were performed by the same surgeon using a medial parapatella approach. To increase articulation curvature during flexion, an additional $2 \mathrm{~mm}$ posterior femoral bone cut was performed. The anterior portion of the tibial component was removed to avoid interference with the patella and the posterior cam mechanism was modified to facilitate high flexion.

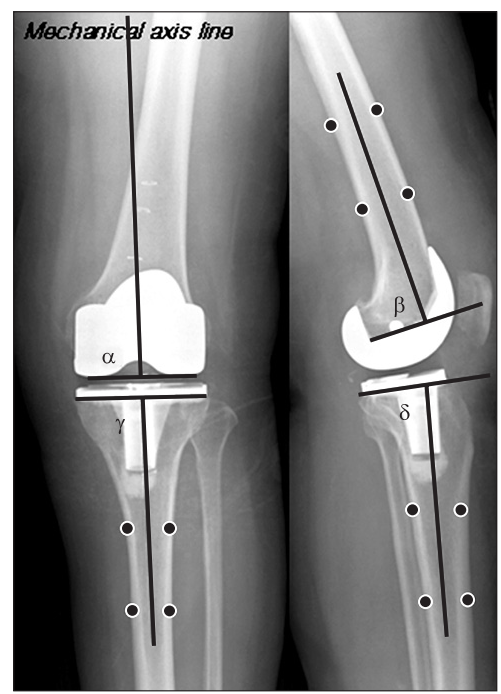

Fig. 2. Implant position angle ( $\alpha$ : coronal medial inclination of femoral component, $\beta$ : sagittal posterior slope of femoral component, $\gamma$ : coronal medial inclination of tibial component, $\delta$ : sagittal posterior slope of tibial component).
The preoperative and postoperative mechanical axes and extent of bone resection could be evaluated using an electromagnetic navigation system in all cases. In particular, varus/valgus femoral resection, degree of flexion/extension, varus/valgus tibial resection, and posterior tilt could be assessed in real time during bone resection of the distal femur and the proximal tibia by placing a paddle on the resection surface (Fig. 3). After bone resection, lower limb alignment was assessed using the navigation system with a temporary implant or an inserted spacer. After implant fixation, the alignment was evaluated and corrected intraoperatively if needed.

For the LPS-Flex Mobile bearing knee, any osteophytes that could cause soft tissue tension were thoroughly removed and subperiosteal release of the medial and lateral collateral ligaments at their insertion sites was performed. Flexion/extension gaps were measured intraoperatively using a gapper in several trial tests to confirm mediolateral stability prior to soft tissue balancing. For the LPS-Flex Mobile bearing knee, tibial resection preceded femoral resection, whereas it was vice versa for the LPSFlex Fixed bearing knee.

\section{Rehabilitation}

Quadriceps femoris strengthening exercises were initiated immediately after surgery. From the 2 nd postoperative day, active joint movement exercises were performed in tandem with passive exercises using CPM devices to facilitate rapid ROM recovery. Weight-bearing ambulation was allowed after suture removal at
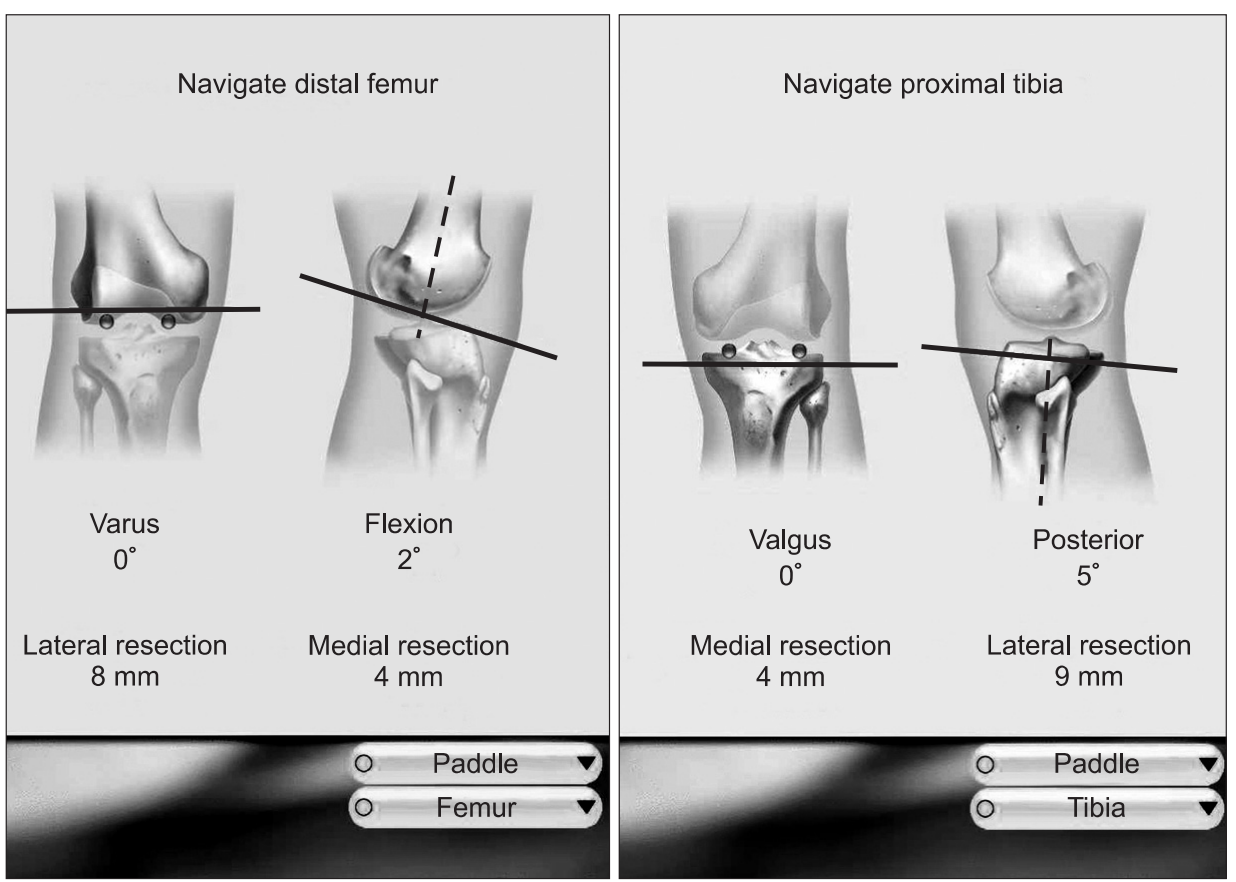

Fig. 3. A display screen in the electomagnetic navigation during resection of the distal femur and proximal tibia (real time information). 
the 2 nd postoperative week.

\section{Statistical Analysis}

Statistical analysis was performed using SPSS ver. 12.0 (SPSS, Inc., Chicago, IL, USA). KSKS, KSFS, WOMAC score, VAS for pain, and ROM and maximal flexion angle before surgery and at the last follow-up were analyzed using an independent sample t-test. Patient ability to kneel and sit cross-legged was assessed using the chi-square test.

\section{Results}

\section{Clinical Results}

There were no statistically significant intergroup differences in the KSKS and KSFS ( $>>0.05)$. In the mobile-bearing group, the mean KSKS and KSFS increased from $48.2(48.2 \pm 6.4)$ preoperatively to $94.5(94.5 \pm 3.2)$ at the last follow-up and from $45.3(45.3 \pm 5.8)$ preoperatively to $93.8(93.8 \pm 2.8)$ at the last follow-up, respectively. In the fixed-bearing group, the mean KSKS and KSFS increased from 49.5 (49.5 \pm 5.6$)$ preoperatively to $95.1(95.1 \pm 2.8)$ at the last follow-up and from $46.9(46.9 \pm 5.9)$ preoperatively to $94.2(94.2 \pm 3.0)$ at the last follow-up ( $p>0.05)$. The mean WOMAC score decreased in both groups, from 81.0 (81.0 \pm 5.5$)$ preoperatively to $14.5(14.5 \pm 1.6)$ at the last followup in the mobile-bearing group and from $82.5(82.5 \pm 6.0)$

Table 2. Clinical results according to KSKS, KSFS, and VAS

\begin{tabular}{|c|c|c|c|c|c|}
\hline & \multicolumn{2}{|c|}{ LPS-flex mobile } & \multicolumn{2}{|c|}{ LPS-flex fixed } & \multirow[b]{2}{*}{ p-value } \\
\hline & Preoperative & $\begin{array}{l}\text { at follow- } \\
\text { up }\end{array}$ & Preoperative & $\begin{array}{l}\text { at follow- } \\
\text { up }\end{array}$ & \\
\hline KSKS & $48.2 \pm 6.4$ & $94.5 \pm 3.2$ & $49.5 \pm 5.6$ & $95.1 \pm 2.8$ & $>0.05$ \\
\hline KSFS & $45.3 \pm 5.8$ & $93.8 \pm 2.8$ & $46.9 \pm 5.9$ & $94.2 \pm 3.0$ & $>0.05$ \\
\hline WOMAC & $81.0 \pm 5.5$ & $14.5 \pm 1.6$ & $82.5 \pm 6.0$ & $15.2 \pm 1.8$ & $>0.05$ \\
\hline VAS & $7.8 \pm 1.6$ & $1.5 \pm 0.6$ & $7.6 \pm 1.5$ & $1.4 \pm 0.5$ & $>0.05$ \\
\hline
\end{tabular}

KSKS: Knee Society Knee Score, KSFS: Knee Society Functional Score, VAS: Visual Analogue Scale, WOMAC: Western Ontario and McMaster Universities Arthritis Index.

Table 3. Daily Living Results from the Possibility of the Kneeling Position and Cross-Legged Sitting Position

\begin{tabular}{lccc}
\hline & LPS-flex mobile & LPS-flex fixed & p-value \\
\hline Case & 32 & 34 & \\
Kneeling & $22(69)$ & $24(71)$ & $>0.05$ \\
Cross legged & $28(88)$ & $29(85)$ & $>0.05$ \\
\hline
\end{tabular}

Values are presented as number (\%). preoperatively to $15.2(15.2 \pm 1.8)$ at the last follow-up in the fixed-bearing group $(\mathrm{p}>0.05)$. There were no notable intergroup differences in the mean VAS pain score ( $p>0.05)$ (Table 2). The mean VAS pain score improved from $7.8(7.8 \pm 1.6)$ preoperatively to $1.5(1.5 \pm 0.6)$ at the last follow-up in the mobile-bearing group and from $7.6(7.6 \pm 1.5)$ preoperatively to $1.4(1.4 \pm 0.5)$ at the last follow-up in the fixed-bearing group.

\section{Kneeling and Cross-Legged Sitting}

Significant intergroup differences were not identified in the ability to kneel and sit cross-legged through observation or interview during the follow-up ( $>>0.05)$ (Table 3). Kneeling and cross-legged sitting was possible in 22 and 28 cases, respectively, in the mobile bearing group ( $\mathrm{n}=32)$ and in 24 and 29 cases, respectively, in the fixed-bearing group ( $\mathrm{n}=34)$.

No remarkable intergroup differences were noted in patients with $\geq 130^{\circ}$ maximal flexion angle (high flexion) ( $p>0.05$ ) (Table 4). The number of patients with $\geq 130^{\circ}$ maximal flexion angle was 18 in the mobile-bearing group and 19 in the fixed-bearing group. Kneeling and cross-legged sitting was possible in 15 and 18 cases, respectively, in patients with high flexion in the mobilebearing group and in 16 and 18 cases, respectively, in patients with high flexion in the fixed-bearing group.

\section{Radiographic Results}

On the lower limb alignment, the mechanical axis deviation was more varus in the fixed-bearing group $\left(0.9^{\circ} \pm 1.1^{\circ}\right)$ than in the mobile-bearing group $\left(0.7^{\circ} \pm 1.0^{\circ}\right)$, but there was no significant difference between the groups. The coronal inclination of femoral component coronal and tibial component was $89.3^{\circ} \pm 2.8^{\circ}$ and

Table 4. Daily Living Results from the Possibility of the Kneeling Position and Cross-legged Sitting Position in MFA Over $130^{\circ}$ Groups

\begin{tabular}{lccc}
\hline & LPS-flex mobile & LPS-flex fixed & p-value \\
\hline Case & $18(56)$ & $19(56)$ & \\
Kneeling & $15(83)$ & $16(84)$ & $>0.05$ \\
Cross legged & $18(100)$ & $18(95)$ & $>0.05$ \\
\hline
\end{tabular}

Values are presented as number (\%).

MFA: maximal flexion angle.

Table 5. Radiologic Results of Limb Alignment after Total Knee Arthroplasty

\begin{tabular}{lccc}
\hline & LPS-flex mobile & LPS-flex fixed & p-value \\
\hline Mechanical axis deviation $\left(^{\circ}\right)$ & $0.7 \pm 1.0$ & $0.9 \pm 1.1$ & $>0.05$ \\
Anatomical axis $\left({ }^{\circ}\right)$ & $5.5 \pm 4.4$ & $5.4 \pm 4.6$ & $>0.05$ \\
\hline
\end{tabular}


Table 6. Radiologic Results of Implant Position after Total Knee Arthroplasty

\begin{tabular}{|c|c|c|c|c|c|}
\hline & \multicolumn{2}{|c|}{ LPS-flex mobile } & \multicolumn{2}{|c|}{ LPS-flex fixed } & \multirow{2}{*}{ p-value } \\
\hline & Femoral component & Tibial component & Femoral component & Tibial component & \\
\hline Coronal plane $\left(^{\circ}\right)$ & $89.3 \pm 2.8$ & $89.8 \pm 1.0$ & $89.6 \pm 3.2$ & $89.3 \pm 1.2$ & $>0.05$ \\
\hline Sagittal plane $\left({ }^{\circ}\right)$ & $88.5 \pm 1.5$ & $86.6 \pm 1.4$ & $88.5 \pm 1.8$ & $86.2 \pm 2.0$ & $>0.05$ \\
\hline
\end{tabular}

Table 7. Radiologic Results of the Range of Motion (ROM) and Maximal Flexion Angle (MFA)

\begin{tabular}{|c|c|c|c|c|c|}
\hline & \multicolumn{2}{|c|}{ LPS-flex mobile } & \multicolumn{2}{|c|}{ LPS-flex fixed } & \multirow{2}{*}{$\mathrm{p}$-value } \\
\hline & Preoperative & At follow-up & Preoperative & At follow-up & \\
\hline ROM & $118.5 \pm 5.8$ & $130.5 \pm 3.8$ & $118.0 \pm 5.2$ & $129.5 \pm 4.0$ & $>0.05$ \\
\hline MFA & $122.0 \pm 5.2$ & $131.3 \pm 3.6$ & $121.8 \pm 5.0$ & $130.8 \pm 3.3$ & $>0.05$ \\
\hline
\end{tabular}
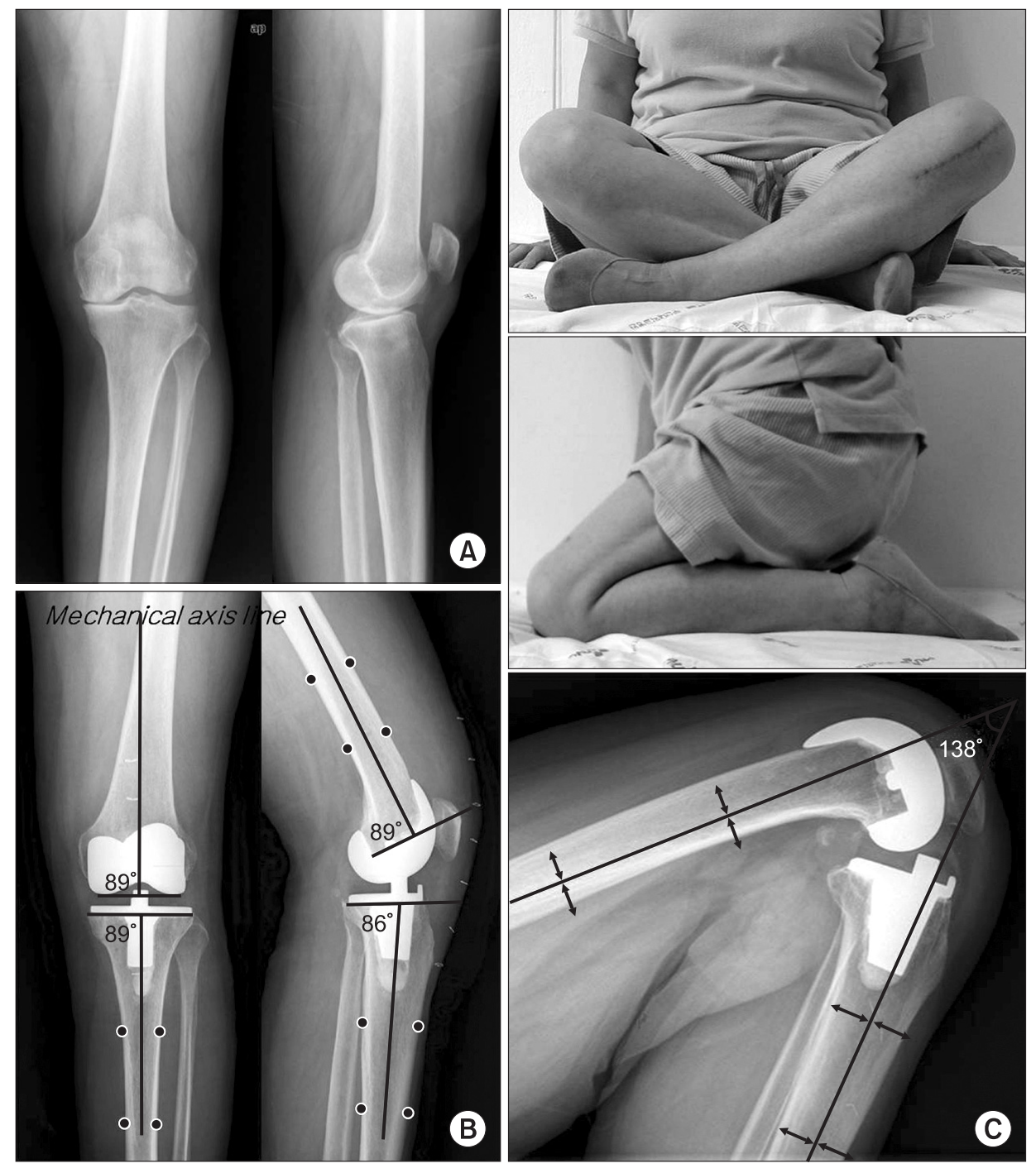

Fig. 4. A 66-year-old woman visited our clinic with pain in both knees for 7 years (A). We performed left total knee arthroplasty using Zimmer LPS-Flex Mobile (B). At the last follow up (14 months after total knee arthroplasty), flexion contracture of left knee joint was $0^{\circ}$, and KSKS was 96 points, which was 50 points preoperatively, and KSFS was 95 points, which was 48 points preoperatively, and WOMAC score was 12 points, which was 85 points preoperatively. Maximal flexion angle of left knee joint was $138^{\circ}$, which was $120^{\circ}$ preoperatively, and cross-legged sitting position and kneeling position were all possible (C). KSKS: Knee Society Knee Score, KSFS: Knee Society Functional Score, WOMAC: Western Ontario and McMaster Universities Arthritis Index. $89.8^{\circ} \pm 1.0^{\circ}$, respectively, in the fixed-bearing group and $89.6^{\circ} \pm 3.2^{\circ}$ and $89.3^{\circ} \pm 1.2^{\circ}$, respectively, in the mobile-bearing group. The sagittal inclination of femoral component and tibial component was $88.5^{\circ} \pm 1.5^{\circ}$ and $86.6^{\circ} \pm 1.4^{\circ}$ in the fixed-bearing group and $88.5^{\circ} \pm 1.8^{\circ}$ and $86.2^{\circ} \pm 2.0^{\circ}$ in the mobile-bearing group. Thus, the coronal and sagittal implant positioning was satisfactory in 

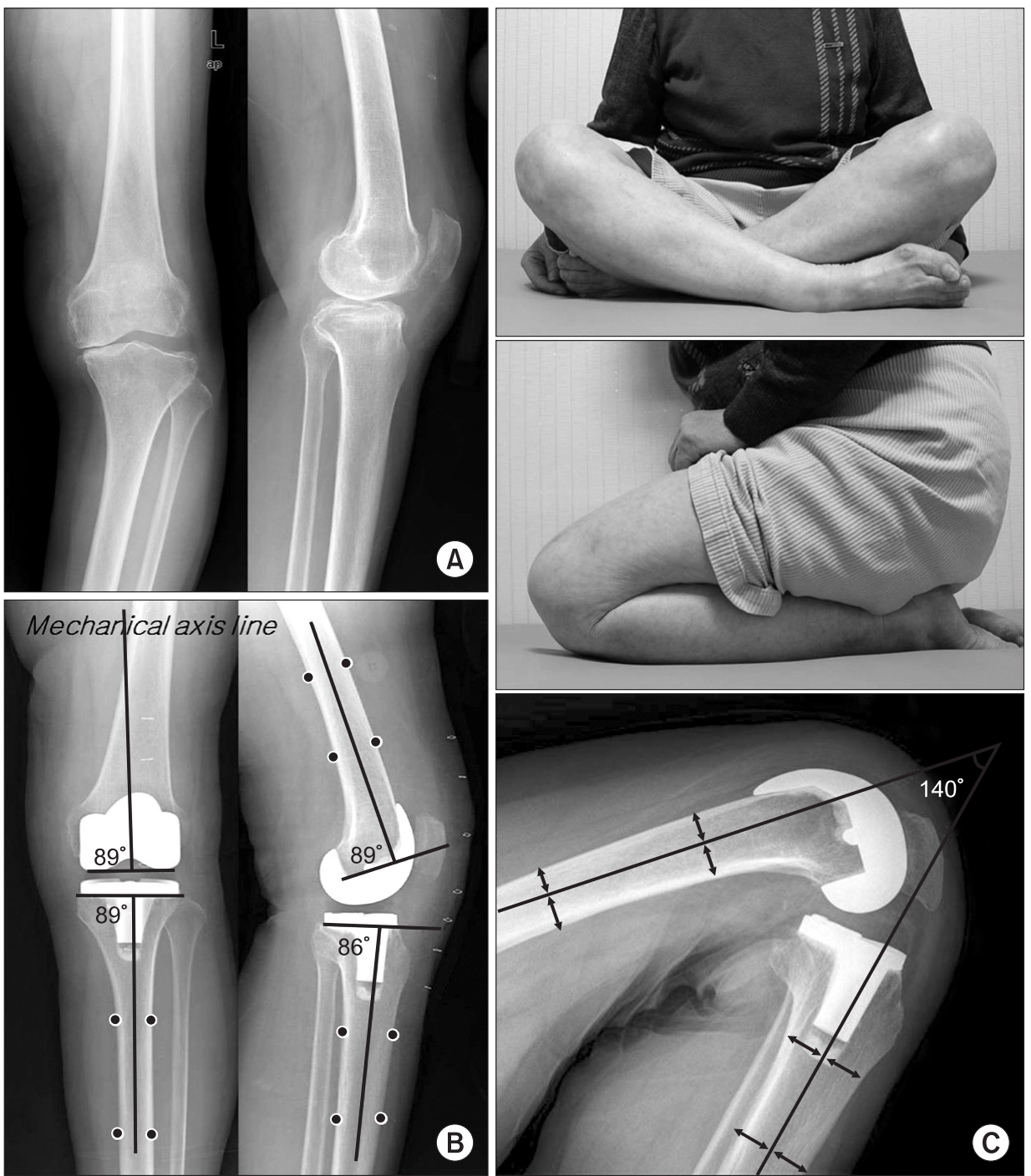

Fig. 5. A 77-year-old woman visited our clinic with both knee pain for 7 years (A). We performed left total knee arthroplasty using Zimmer LPS-Flex Fixed (B). At the last follow-up (13 months after total knee arthroplasty), flexion contracture of left knee joint was $0^{\circ}$, and KSKS was 94 points, which was 49 points preoperatively, and KSFS was 95 points, which was 51 points preoperatively, and WOMAC score was 13 points, which was 84 points preoperatively. Maximal flexion angle of left knee joint was $140^{\circ}$, which was $119^{\circ}$ preoperatively, and cross-legged sitting position and kneeling position were all possible (C). KSKS: Knee Society Knee Score, KSFS: Knee Society Functional Score, WOMAC: Western Ontario and McMaster Universities Arthritis Index. both groups, which showed no significant intergroup differences (Tables 5, 6).

\section{ROM and Maximum Knee Flexion}

There were no statistically significant intergroup differences in ROM and maximal flexion angle ( $\mathrm{p}>0.05)$. The mean ROM increased from $118.5(118.5 \pm 5.8)$ preoperatively to 130.0 (130.0 $\pm 3.8)$ at the last follow-up in the mobile-bearing group and from $118.0(118.0 \pm 5.2)$ preoperatively to $129.5(129.5 \pm 4.0)$ at the last follow-up in the fixed-bearing group. The mean maximal flexion angle increased from $122.0(122.0 \pm 5.2)$ preoperatively to 131.1 $(131.1 \pm 3.6)$ at the last follow-up in the mobile-bearing group and from $121.8(121.8 \pm 5.0)$ preoperatively to $130.8(130.8 \pm 3.3)$ at the last follow-up in the fixed-bearing group ( $\mathrm{p}>0.05)$ (Table 7 ) (Figs. 4, 5).

\section{Complications}

Polyethylene component wear, posterior instability, component loosening, or infection was not observed in any of the patients during the $\geq 1$ year follow-up period. Complications requiring revision surgery were not noted during the short-term follow-up period.

\section{Discussion}

The primary goal of TKA is to alleviate pain but it is more favorable to restore normal knee function ${ }^{8)}$. Postoperative ROM is especially important for Asian patients who frequently squat or sit in a cross-legged position".

Goodfellow and O'Connor ${ }^{10)}$ introduced a mobile-bearing knee system in 1976 to address component loosening and wear, the two most common issues of TKA. High flexion knee 
system is a recent invention for overcoming ROM restriction. The mechanism that hinders knee flexion remains unclear, but the ability to restore posterior femoral translation has been considered crucial for improving range of flexion after TKA ${ }^{11,12}$. A decrease in posterior femoral translation causes impingement of the posterior edge of the tibial component on the femoral shaft, thus constraining flexion motion ${ }^{11-13)}$. High flexion knee system was designed to increase posterior femoral translation for high flexion ${ }^{12,13)}$.

We used the high-flexion LPS-Flex total knee system in this study. An additional $2 \mathrm{~mm}$ posterior femoral bone resection was performed to extend the posterior condyle of the femoral component by $2 \mathrm{~mm}$ and increase the articular contact area and thus posterior femoral translation for high flexion. In addition, the stress on the polyethylene implant was reduced, which contributed to the low incidence of component wearing. The LPS-Flex Mobile system is a mobile bearing implant designed to allow for a total of $50^{\circ}$ of axial rotation (internal rotation, $25^{\circ}$ and external rotation, $25^{\circ}$ ) of the polyethylene insert in the tibial component during knee flexion. Therefore, we used a technique similar to the 'gap technique' for soft tissue balancing in the mobile-bearing group: after tibial resection, internal/external stability was confirmed by measuring the flexion/extension gaps in several trial tests. In contrast, 'measured resection technique' where femoral resection precedes tibial resection, was used in the fixed-bearing group. In our opinion, comparisons of the postoperative lower limb alignment and coronal/sagittal implant positions between the groups should be performed in further studies.

A navigation system was used to improve accuracy of TKA in this study. Therefore, a range of errors could be identified at every step of the surgery and the surgeon could give real time feedback based on surgical experience and intraoperative data to achieve ideal values ${ }^{14}$. There are many studies showing that navigation-assisted TKA produces more satisfactory results than the established TKA in terms of implant positioning and lower limb alignment that are associated with long-term longevity ${ }^{15-18)}$.

Mobile-bearing knee prostheses are more effective in reducing fatigue wear because the enlarged contact surface causes less stresses compared to fixed-bearing ones ${ }^{19)}$ and in lowering stresses on the bone-implant interface and risk of loosening, which potentially increases implant longevity ${ }^{10,20)}$. Another advantage is that the motion between the tibial component and the polyethylene insert can be adjusted to minimize backside wear ${ }^{21)}$.

Clinical results of TKA using mobile-bearing and fixed-bearing implants have been addressed in various studies. Woolson et al. ${ }^{22)}$ reported that there were no significant differences between the mobile-bearing implant group (57 cases) and fixed-bearing implant group (45 cases) in terms of the KSKS, KSFS, pain score, and postoperative flexion angle. Ranawat et al. ${ }^{23)}$ used mobilebearing and fixed-bearing implants during TKA in 26 patients and found no notable differences between the two implants in terms of the KSKS and KSFS, postoperative ROM and maximal flexion angle, knee pain, and patient satisfaction. Lampe et al. ${ }^{24)}$ used navigation in 52 cases of mobile-bearing knee replacement and 48 cases of fixed-bearing knee replacement. At $\geq 2$ years postoperatively, no significant differences were noted in the KSKS and KSFS, Oxford knee score, and ROM. Kim et al. ${ }^{25}$ performed 116 cases of bilateral TKAs using a mobile-bearing implant and a fixed-bearing implant on each side and found no significant differences between the two types of implants in terms of the ROM, KSKS, pain score, patient satisfaction, and polyethylene wear.

In contrast, Price et al. ${ }^{26)}$ reported that the KSKS and pain score at 1 year after surgery were higher, albeit slightly, in the mobilebearing group although the ROM was similar between the groups with $105.3^{\circ}$. Luring et al..$^{27)}$ reported that the mediolateral stability was higher in the mobile-bearing group after PCL retaining TKA. Sohn et al. ${ }^{28)}$ followed 32 patients that underwent bilateral TKA using a mobile-bearing implant and a fixed-bearing implant on each side and noted that the postoperative subjective satisfaction and preference evaluated using the WOMAC score was higher in the mobile-bearing group although the two implants did not result in differences in the KSKS, KSFS, and postoperative ROM.

In this study, significant intergroup differences were not observed in the KSKS and KSFS, WOMAC score, ROM and maximal flexion angle, and possibility of kneeling and sitting cross-legged $(p>0.05)$. The radiographic results showed that there were no notable intergroup differences in the postoperative mechanical axis deviation and the femoral and tibial component positions in the sagittal and coronal planes $(p>0.05)$. However, we think these results should be confirmed by further studies with longer follow-up periods.

\section{Conclusions}

The clinical results of high-flexion TKA were satisfactory in both the mobile-bearing and fixed-bearing group. There were no significant intergroup differences regarding the clinical results (KSS, KSFS, and WOMAC score), radiological results (ROM and maximal flexion angle), and possibility of kneeling and sitting 
cross-legged. However, these results should be verified in longterm follow-up studies that address the longevity of the implants.

\section{References}

1. Harvey IA, Barry K, Kirby SP, Johnson R, Elloy MA. Factors affecting the range of movement of total knee arthroplasty. J Bone Joint Surg Br. 1993;75:950-5.

2. Matsumoto T, Muratsu H, Tsumura N, Mizuno K, Kuroda $\mathrm{R}$, Yoshiya S, Kurosaka M. Joint gap kinematics in posteriorstabilized total knee arthroplasty measured by a new tensor with the navigation system. J Biomech Eng. 2006;128:86771.

3. Lizaur A, Marco L, Cebrian R. Preoperative factors influencing the range of movement after total knee arthroplasty for severe osteoarthritis. J Bone Joint Surg Br. 1997;79:626-9.

4. Stern SH, Insall JN. Total knee arthroplasty in obese patients. J Bone Joint Surg Am. 1990;72:1400-4.

5. Bellamy N. Pain assessment in osteoarthritis: experience with the WOMAC osteoarthritis index. Semin Arthritis Rheum. 1989;18:14-7.

6. Edwards JZ, Greene KA, Davis RS, Kovacik MW, Noe DA, Askew MJ. Measuring flexion in knee arthroplasty patients. J Arthroplasty. 2004;19:369-72.

7. Seon JK, Song EK. The accuracy of lower extremity alignment in a total knee arthroplasty using computerassisted navigation system. J Korean Orthop Assoc. 2004;39:566-71.

8. Cho WS, Park JH, Kim JM, Hwang WY, Nam TS. Factors affecting range of motion after total knee arthroplasty. J Korean Orthop Assoc. 2003;38:683-8.

9. Ritter MA, Campbell ED. Effect of range of motion on the success of a total knee arthroplasty. J Arthroplasty. 1987;2:957.

10. Goodfellow JW, O'Connor J. Clinical results of the Oxford knee. Surface arthroplasty of the tibiofemoral joint with a meniscal bearing prosthesis. Clin Orthop Relat Res. 1986;(205):21-42.

11. Li G, Schule SL, Zyontz SJ. Maloney WJ, Rubash HE. Improving flexion in total knee arthroplasty. In: Callaghan JJ, Rosenberg AG, Rubash HE, Simonian PT, Wickiowicz TL, editors. Vol. 2. Philadelphia: Lippincott Williams and Wilkins; 2003. p.1233-4.

12. Walker PS, Garg A. Range of motion in total knee arthroplasty. A computer analysis. Clin Orthop Relat Res.
1991;(262):227-35.

13. Song YJ, Ha CW. Functional evaluation of high-flex total knee arthroplasty. J Korean Knee Soc. 2009;21:29-34.

14. Kanlic EM, Delarosa F, Pirela-Cruz M. Computer assisted orthopaedic surgery: CAOS. Bosn J Basic Med Sci. 2006;6:713.

15. Ensini A, Catani F, Leardini A, Romagnoli M, Giannini S. Alignments and clinical results in conventional and navigated total knee arthroplasty. Clin Orthop Relat Res. 2007;457:156-62.

16. Anderson KC, Buehler KC, Markel DC. Computer assisted navigation in total knee arthroplasty: comparison with conventional methods. J Arthroplasty. 2005;20:132-8.

17. Bathis H, Perlick L, Tingart M, Luring C, Zurakowski D, Grifka J. Alignment in total knee arthroplasty. A comparison of computer-assisted surgery with the conventional technique. J Bone Joint Surg Br. 2004;86:682-7.

18. Chin PL, Yang KY, Yeo SJ, Lo NN. Randomized control trial comparing radiographic total knee arthroplasty implant placement using computer navigation versus conventional technique. J Arthroplasty. 2005;20:618-26.

19. Otto JK, Callaghan JJ, Brown TD. Gait cycle finite element comparison of rotating-platform total knee designs. Clin Orthop Relat Res. 2003;(410):181-8.

20. Lee DC, Sohn OJ. A comparison of mobile-bearing and fixed-bearing total knee arthroplasties in osteoarthritic patients. J Korean Knee Soc. 2007;19:154-60.

21. Callaghan JJ, Squire MW, Goetz DD, Sullivan PM, Johnston RC. Cemented rotating-platform total knee replacement. A nine to twelve-year follow-up study. J Bone Joint Surg Am. 2000;82:705-11.

22. Woolson ST, Epstein NJ, Huddleston JI. Long-Term Comparison of Mobile-Bearing vs Fixed-Bearing Total Knee Arthroplasty. J Arthroplasty. 2011;26:1219-23.

23. Ranawat AS, Rossi R, Loreti I, Rasquinha VJ, Rodriguez JA, Ranawat CS. Comparison of the PFC Sigma fixed-bearing and rotating-platform total knee arthroplasty in the same patient: short-term results. J Arthroplasty. 2004;19:35-9.

24. Lampe F, Sufi-Siavach A, Bohlen KE, Hille E, Dries SP. One year after navigated total knee replacement, no clinically relevant difference found between fixed bearing and mobile bearing knee replacement in a double-blind randomized controlled trial. Open Orthop J. 2011;5:201-8.

25. Kim YH, Kook HK, Kim JS. Comparison of fixed-bearing and mobile-bearing total knee arthroplasties. Clin Orthop Relat Res. 2001;(392):101-15. 
26. Price AJ, Rees JL, Beard D, Juszczak E, Carter S, White S, de Steiger R, Dodd CA, Gibbons M, McLardy-Smith P, Goodfellow JW, Murray DW. A mobile-bearing total knee prosthesis compared with a fixed-bearing prosthesis. A multicentre single-blind randomised controlled trial. J Bone Joint Surg Br. 2003;85:62-7.

27. Luring C, Bathis H, Oczipka F, Trepte C, Lufen H, Perlick L,
Grifka J. Two-year follow-up on joint stability and muscular function comparing rotating versus fixed bearing TKR. Knee Surg Sports Traumatol Arthrosc. 2006;14:605-11.

28. Sohn OJ, Lee DC, Cho JH. Comparision between mobile bearing and fixed bearing T.K.A. in the same patient. J Korean Orthop Assoc. 2008;43:200-6. 